\title{
The Road to Success: Recommendations for the Design of Successful Business Process Modeling Initiatives
}

\author{
Marcus Fischer \\ University of Wuerzburg \\ marcus.fischer@uni-wuerzburg.de
}

Christian Janiesch

University of Wuerzburg

christian.janiesch@uni-wuerzburg.de

\author{
Florian Imgrund \\ University of Wuerzburg \\ florian.imgrund@uni-wuerzburg.de
}

\author{
Axel Winkelmann \\ University of Wuerzburg \\ axel.winkelmann@uni-wuerzburg.de
}

\author{
Julian Kolb \\ University of Wuerzburg \\ julian.kolb@uni-wuerzburg.de \\ Christoph Rosenkranz \\ University of Cologne \\ rosenkranz@wiso.uni-koeln.de
}

\begin{abstract}
Process modeling is among the most important activities in the business process management lifecycle and enables enterprises to improve communication, coordination, and knowledge management. However, enterprises frequently face challenges when introducing process modeling to their organization. These range from a lack of strategic alignment to insufficient stakeholder participation leading to pitfalls, such as project failure or outcomes that do not meet predefined expectations. Hence, we present findings from eight successful process modeling initiatives and consolidate them to a decision-support framework. The contribution of this research is twofold. First, our study suggests that the success of process modeling depends on contextual parameters, including top management involvement, tool support, and employee education. Second, the design of process modeling initiatives fundamentally changes with organizational objectives. Based on a clear set of goals, enterprises can choose from at least three success strategies that require a unique configuration and structure.
\end{abstract}

\section{Introduction}

In a dynamic business environment, enterprises must constantly adapt and improve operations, consolidate organizational structures, reduce costs, and improve efficiency and effectiveness. In general, they can accomplish this by implementing business process management (BPM) as a means to identify, prioritize, analyze, improve, and monitor business processes [1]. At the core of BPM, process modeling provides the basis for communication, coordination, documentation, and implementation [2].

Although enterprises perform process modeling to accomplish similar goals, for example, to improve operational quality, initiatives in practice can differ regarding their size, design, structure, and organization $[2,3]$. This leads to a "one size does not fit all" situation. While one organization may follow a decentral approach and use process models only for communication and knowledge management, another highly hierarchical organization uses it to implement and automate its core processes. It is only natural that they require a different setup and management of process modeling. Established modeling standards and specifications, however, do not provide support for situating process modeling in a specific business environment. They provide uniform notation symbols and grammar, but no means for contextualization and adaptation. Based on these observations, we summarize our research questions (RQ) as follows:

$$
\begin{aligned}
& \text { (RQ1) Which success factors for process } \\
& \text { modeling in practice can we identify? } \\
& \text { (RQ2) How can enterprises successfully } \\
& \text { establish a process modeling initiative? }
\end{aligned}
$$

To answer these RQ, this paper presents practical insights from eight companies that have established process modeling successfully. We draw upon the Six Core Elements of BPM framework to structure our discussion [1]. We examine each company and show how the design of process modeling initiatives depends on organizational requirements and demands general and contextual capabilities. For enterprises facing such situations in practice, we derive recommendations and a decision-support framework that yields three strategy blueprints for process modeling success.

Our paper is structured as follows: Section 2 describes the applied research method. In Section 3, we present results from a literature analysis and identify a set of success factors for process modeling. We summarize key characteristics of the case companies, describe the applied data collection and analysis procedures, and present case study findings in Section 
4. Subsequently, we derive a management framework in Section 5 and present three success strategies for process modeling in Section 6. We conclude our research with a summary of findings, limitations, and future research opportunities.

\section{Research Method}

First, we analyze the literature based on established guidelines and concepts [4] to develop a success factor model for modeling in practice. We search multiple literature databases for contributions on success factors of process modeling initiatives. We focus on practiceoriented studies, such as case studies or empirical papers, as they link situations faced to actions taken. We adapt the approach of Indulska, et al. [5] to organize our findings.

Second, to obtain empirical insights on how enterprises address these success factors, we employ a case study research design, which is suitable for exploratory research and theory building [6]. To ensure rigorousness, we use an iterative procedure for data collection, analysis, synthesis, and interpretation. Thereby, we sought to avoid potential biases by incorporating as many data sources as possible. To ensure reproducibility, we documented each step of our research. We ensure reliability by building a case collection that contains protocols and supplementary data, such as transcripts, guidelines, modeling conventions, and memos [6]. We use multiple data sources and apply pattern matching to integrate our findings and to ensure construct and predictive validity. Ultimately, we address external validity by following a cross-company research design.

\section{$3 \quad$ Process Modeling Success Factors}

In our first step, we use the approach of Indulska, et al. [5], who draw upon the Six Core Elements of BPM framework to structure issues and challenges of modeling initiatives. We use this framework, because it captures high-level requirements for successful BPM initiatives. We also considered other concepts, such as those proposed by Dumas, et al. [3] or summarized by Röglinger, et al. [7], but found them to be not suitable due to inadequate focus.

In general, the framework distinguishes the six core elements of strategic alignment, governance, methods, information technology, people, and culture [1]. Strategic alignment suggests enterprises to align BPM activities to their organizational priorities. Governance further demands them to ensure accountability, for example, by determining a system of roles and responsibilities. Methods comprise techniques and tools that support activities along the BPM lifecycle, while information technology demands software for process analysis and modeling. People create, enhance, apply, or refuse BPM outcomes. Ultimately, culture demands collective beliefs toward a process-centered organization.

Similar to BPM, process modeling itself constitutes an organizational change process that requires enterprises to define goals, formulate a strategy, and establish different organizational capabilities [5]. Subsequently, we use the dimensions of the framework to build a success factor model for process modeling.

The area of strategic alignment comprises the success factors alignment, architecture, and communication. Due to scarce resources, enterprises must link modeling to strategical priorities [3]. To deploy resources effectively, they must further provide a process architecture as a means to coordinate process modeling activities [8]. While various studies highlight the positive impact of process modeling on enterprise performance, the task itself remains complex and time consuming [8]. Indulska, et al. [5] emphasize that stakeholders do not perceive modeling benefits automatically. Instead, enterprises must communicate them continuously. They further rely on a guiding strategy, which should address various aspects, such as process prioritization and communication [5, 9].

The core element of Governance includes the success factors of specifications and responsibilities. Process modeling requires enterprises to define rules on whether a process is modeled or not. As governance describes a major challenge in most process modeling projects [5], enterprises must provide a clear set of roles, responsibilities, and modeling standards. These can include ontologies, process templates, coordination strategies, and guidelines for quality assurance, exception handling, rewards, and remuneration structures [10, 11]. Hampering the comparability of process models, the task of selecting an adequate abstraction level constitutes a main success factor in modeling initiatives [5, 8]. Although standardization enables communication, it can reduce feasibility in specialized areas. Furthermore, several authors assert an increasing erosion of modeling standards, the emergence of dialects, and the use of extensions or proprietary standards [12]. Enterprises must address the tradeoff between standardization and flexibility by analyzing their business context, defining adequate conventions and guidelines, and selecting a subset of modeling instruments [8]. Thus, they can realize benefits from standardization, while providing flexibility in special business scenarios [13].

The core element of methods comprises the success factors of language, collaboration, and version and 
variant management. Enterprises must support their modeling activities with adequate techniques and languages. In the past, many such techniques have been introduced [14, 15]. However, Green and Rosemann [16] observe a growing complexity, which results from representational deficiencies. In general, Recker, et al. [14] distinguish between construct deficits, redundancies, excesses, and overloads. Recker, et al. [17] further points out that most notations lack clarity and understandability, which negatively affects their perceived usability and usefulness. For Business Process Modeling and Notation (BPMN) especially, Recker [18] describes a lack of support for decomposition and organizational modeling. Thus, enterprises must select a notation that complies with organizational and user requirements. If adaptations are necessary, Rosemann [13] recommends to focus on aspects that yield significant improvements in usability and applicability. Besides notational issues, challenges result from the management of versions, variants, and releases [19]. As modeling is increasingly performed collaboratively, stakeholders produce large repositories $[5,20]$. Enterprises rely on mechanisms to structure their process knowledge.

The core element of information technology comprises the success factors of tool support, integration, and collaboration platform. In particular, it provides enterprises with the means to increase the efficiency, consistency, and quality of process modeling [5]. Tools frequently cover multiple application scenarios, including process mining, process automation, and process simulation [21, 22]. To facilitate software usage, Rosemann [8] suggests to evaluate candidate tools and vendors toward their capabilities to meet organizational requirements. Modeling should further not stay an end to itself, but connect with other software or a process execution engine [23]. However, if enterprises seek to realize benefits from automation, they rely on mechanisms for transforming process models into machine-readable process code [23]. Ultimately, modeling initiatives can involve multiple stakeholders with different objectives and viewpoints. To streamline their distributed activities, enterprises rely on a central platform that facilitates communication and collaboration [8, 24, 25]. The core element of people further requires enterprises to address the success factors of training and ease of use. As people apply, refine, and enhance process models, they are essential for successful modeling initiatives. Due to the task's scope and complexity, several authors notice that modelers frequently lack necessary skills and expertise [5]. In fact, process modeling demands knowledge of software tools, experience in leading workshops and interviews, and expertise in setting up understandable representations from comments or documentations [8]. To establish these capabilities, enterprises rely on an education program that conciliates conceptual and practical competencies. To foster communication and collaboration, they should further equip their platform with functionalities for accessing and sharing process knowledge and modeling outcomes [26]. Hence, they can realize synergy effects and support stakeholders in reusing available knowledge [26]. To improve model applicability, enterprises must determine a degree of complexity, which enables them to capture details without exceeding stakeholder capabilities. Thus, they rely on easy-to-use methods and tools [27, 28].

In the area of culture, enterprises must address the success factors of stakeholder commitment and process awareness. They rely on collective values and a process-oriented environment that encourages stakeholders to participate [5, 29]. Thus, they must ensure the buy-in and commitment of relevant stakeholders [5, 13]. As process models can be accessed over multiple channels, employees typically analyze and apply them without additional support from experts. To support them in doing so productively, enterprises must weight syntactic and semantic quality with modeling intuitiveness [27]. In the past, enterprises were shaped by functional structures. However, successful modeling initiatives require process orientation. Hence, they must communicate benefits of process awareness and add related aspects to their educational programs [5, 29].

We summarize the success factor model in Figure 1 and thereby answer RQ1.

\begin{tabular}{|c|c|c|c|c|c|}
\hline \multicolumn{5}{|c|}{ Core Elements } \\
\hline $\begin{array}{c}\text { Strategic } \\
\text { Alignment }\end{array}$ & Governance & Methods & $\begin{array}{c}\text { Information } \\
\text { Technology }\end{array}$ & People & Culture \\
\hline \hline \multicolumn{5}{|c|}{ Success Factors for Process Modeling } \\
\hline \hline Strategy & Specifications & Languages & Tool Support & Training & Commitment \\
\hline \hline Architecture & Responsibilities & Collaboration & Integration & Ease of Use & $\begin{array}{c}\text { Process } \\
\text { Awareness }\end{array}$ \\
\hline \hline Communication & & $\begin{array}{c}\text { Versions \& } \\
\text { Variants }\end{array}$ & $\begin{array}{c}\text { Collaboration } \\
\text { Platform }\end{array}$ & \\
\hline \hline & & & \\
\end{tabular}

Figure 1. Process modeling success factors

\section{$4 \quad$ Case Study Results}

We conducted eight case studies in seven large German and Australian companies to analyze how to successfully address the factors of our model. To produce generalizable implications, we include companies that differ in size, business focus, industry, and strategy. However, each company has established process modeling successfully. We summarize the companies' key characteristics in Table 1. 
Table 1. Characteristics of the case companies

\begin{tabular}{lccc}
\hline & Interviews & Industry & Location \\
\hline Company $1(\mathrm{Cl})$ & 2 & Enterprise Software & Australia \\
Company $2(\mathrm{C} 2)$ & 1 & Public & Australia \\
Company $3(\mathrm{C3})$ & 1 & Railway Operator & Australia \\
Company $4(\mathrm{C4})$ & 1 & Public & Australia \\
Company $5(\mathrm{C5})$ & 1 & Public & Australia \\
Company $6(\mathrm{C6})$ & 2 & Carrier & Germany \\
Company $7(\mathrm{C} 7)$ & 1 & Food & Germany \\
Company $8(\mathrm{C} 8)$ & 1 & Enterprise Software & Germany \\
\hline
\end{tabular}

$\mathrm{C} 1$ and $\mathrm{C} 8$ are subsidiaries of a leading company for enterprise software, which operates in more than 130 countries and employs over 80,000 people. $C 2$ is an Australian law firm with more than 1,000 employees. The company focuses on the management of intellectual property rights on behalf of the Australian government. C3 provides transport and logistic business solutions to a diverse range of local, regional, and global customers. The company employs over 7,000 people. $C 4$ is a statutory authority that is responsible for collecting and providing land and property information in Western Australia. The company has about 500 employees and offers services, such as certification, property sales reporting, and survey plans. C5 is an Australian institution that manages government investments in information and communication technology. The company provides advice to other government agencies and consults executives on several policy issues. C6 is a German telecommunication company that offers Internet access products, hosting, and cloud-based business solutions. The company employs over 8,000 people and serves more than 9 million customers. Ultimately, C7 is a German company with over 200 employees that produces and distributes natural and vegan food products.

\subsection{Data Collection}

We conduct multiple interviews with BPM managers of the case companies. For each interview, we use identical questionnaires, typically requiring 90 minutes to answer. Our questionnaire comprises three parts: Part A covers the interviewees' background and expertise. Part B addresses their view on and comprehension of process modeling. Ultimately, part C prompts for information about the companies' strategies and the actions taken to address the dimensions of our success factor model. The interviews include both open and closed questions. While we use open questions to discuss broader aspects of modeling, closed questions are asked to gather specific information about the interviewees' beliefs and comprehensions.

\subsection{Case Study Analysis}

We initiate our analysis by building a comprehensive case collection. We further develop a coding scheme to identify patterns and deviations. Ultimately, we integrate our findings to initiate the framework construction procedure and to answer RQ2. In general, all case companies address each area of the success factor model. For reasons of clarity, we focus on the most significant observations.

Strategic Alignment. For strategic alignment, our success factor model suggests enterprises to define a strategy that links modeling to organizational priorities. Enterprises further rely on communication and an architecture that guides and coordinates modeling.

To align modeling with their overall strategy, the case companies recommend defining consistent goals. For example, $\mathrm{C} 2$ uses modeling to improve communication and coordination and thus to increase its operational effectiveness. C3 supports its central reengineering efforts and facilitates process automation and execution. At $\mathrm{C} 6$, modeling provides the means to document implicit knowledge at the place of process execution and to improve communication. $\mathrm{C} 1, \mathrm{C} 5$, and C8 further use it to accomplish standardization. By contrast, $\mathrm{C} 4$ and $\mathrm{C} 7$ focus on improving business IT alignment. Most case companies perform modeling top-down, which demands prioritizing processes for an effective use of organizational resources [3]. C3 prioritizes processes based on process importance and health. Hence, the company assigns dysfunctional or non-performing processes to recurring modeling and improvement cycles. C6 follows a bottom-up approach that does not require process prioritization. Instead, the approach builds upon distributed stakeholders that model their own processes independently. C8 implements modeling as a hybrid approach. Hence, it combines top-down defined priorities with IT requirements that are collected in a bottom-up manner.

Regarding communication, $\mathrm{C} 1$ noticed that successful modeling depends on its perceived benefits, usefulness, and relevancy. C3 and C5 deploy measures to establish modeling as an essential part of working routines and procedures. C6 follows a collaborative approach in which it facilitates stakeholder participation by emphasizing the project's importance for company success. Communication is further complemented with a top management that openly approves process modeling. At most companies, top management takes on an active role within the initiative. This includes monitoring and coordinating activities as well as performing decision making. At 
$\mathrm{C} 8$, the management requests periodic meetings for updates on achievements and challenges that have occurred during the initiative. The management of C6 focuses on providing resources, without becoming actively involved. It is not perceived as a controlling instance, as this hampers stakeholder participation.

All case companies further use process architectures to structure their operations and to determine the scope of their initiatives. $\mathrm{C} 1$ defines different architectures for different parts of the organization. C2, C3, C5, C6, and C8 use multi-tiered concepts that comprise core processes on upper levels and support processes on lower levels.

Governance. The area of governance requires enterprises to standardize the modeling task and to define specifications that facilitate an adequate outcome quality. Furthermore, they rely on roles and responsibilities that ensure accountability.

All case companies, except for $\mathrm{C} 6$, use modeling specifications that, for example, recommend modelers to use as few elements as possible. C1 uses conventions to foster modeling quality and consistency. Furthermore, the company provides a glossary that supports knowledge management and builds a common understanding of its business context. At C2, C7, and C8, modelers can augment process models with supplementary text fields and comments. All process models are then semantically connected to build an organization-wide ontology. To increase simplicity and readability, modeling at $\mathrm{C} 8$ does not consider decisions and business rules. The company further uses a standardized set of modeling elements and continuously modifies it to address changing requirements. $\mathrm{C} 7$ provides different sets of conventions for different user groups to address individual information demands. When C6 introduced the modeling initiative, the company mostly waived guidelines and conventions to ensure stakeholder participation. However, it realized that this approach did not only result in low-quality modeling outcomes, but also hampered modeling itself, as low-skilled modelers require guidance and support. The company implemented a set of basic and conventions that guide modeling procedures without overcomplicating the task. Due to the company's decentral approach, modeling yields large process repositories. To organize them, it establishes naming conventions and guidelines. Specifications are not enforced actively, as this would hamper stakeholder participation.

$\mathrm{C} 1$ and C8 specify areas of responsibility and use their process architecture to assign roles to stakeholders. Similarly, at C8, governance is linked to process architecture levels. While the company follows a central approach for its core processes, it delegates responsibilities for support processes to departments.
At C2 and C7, governance includes a quality management cycle that checks the syntactic and semantic correctness of process models prior to their release. Initially, C3 followed a central governance approach but noticed several constraints. Today, the company delegates most responsibilities to subsidiaries and branches, but still performs conformance checking and quality management centrally. At C4, governance is based on a hybrid approach with local teams performing initial checkups and a central data administration team reviewing the models subsequently. C6 mostly waives central governance as part of its bottom-up approach. Rather than enforcing conformance with specifications, the central department focuses on monitoring modeling adoption. Governance is mostly deployed by the company's modeling tool, which features many such functionalities, including runtime checks and recommendations. The company further uses data analysis methods to control for patterns or anomalies within its process repository.

Methods. In the area of methods, enterprises must select a modeling language to foster communication. They rely on mechanisms for collaboration as well as for variant and version management.

Regarding modeling languages, all case companies select BPMN for modeling. While most companies use its original version, $\mathrm{C} 8$ adapts its constructs and rules to conform with specific organizational requirements. Although collaboration becomes increasingly relevant, only a few companies refer to it as a success factor. In fact, most companies limit collaboration to providing meta data on processes, for example, on involved stakeholders and their responsibilities. C1 focuses on a small set of interactions, such as passing process models to other stakeholders. At $\mathrm{C} 8$, collaboration is an essential part of modeling. Hence, process analysts identify and question all stakeholders for data collection. To achieve a consistent understanding, they further organize workshops, in which they discuss the results of the data collection phase. To support its bottom-up strategy, collaboration is highly important at C6. Hence, all employees have access to a central tool that features social networking functionalities, including commenting, sharing, and tagging.

Although the management of versions and variants is often mentioned within the interview data, only a few companies confirm its importance for process modeling. $\mathrm{C} 1, \mathrm{C} 2, \mathrm{C} 4$, and $\mathrm{C} 8$ initially arrange their processes within their process architectures and store process models in a central database. Thereby, they draw upon naming conventions for structuration purposes. At $\mathrm{C} 1$ and $\mathrm{C} 6$, process stakeholders can derivate from process models to adapt them to their individual needs. $\mathrm{C} 1$ merges all variants into a holistic 
model, which is then released to the organization. $\mathrm{C} 2$ further implements a version-management system to track and analyze model-specific modifications. At C6, employees access the repository through a collaboration platform, which directly connects to the modeling environment. In addition, C8 manages its repository with indicators for process maturity.

Information Technology. Enterprises rely on tools that support modeling and enable process automation. Collaboration platforms connect stakeholders and facilitates communication and coordination.

The importance of tool support is consistently confirmed across all case companies. C1, C6, and C8 provide an integrated tool that features functionalities for modeling, storing, and organizing processes. C2, $\mathrm{C} 3$, and C4 use a modeling tool to support quality management and to enforce modelers' conformance with conventions and guidelines. At $\mathrm{C} 2$, the modeling tool further supports knowledge management and provides the means to specify roles and responsibilities within processes. At $\mathrm{C} 3$ and $\mathrm{C} 4$, the modeling tool uses meta data of processes to organize the companies' process repositories and to facilitate data accessibility. As C3 initially failed to establish an integrated IT infrastructure, process models were distributed across multiple repositories within the company. Hence, the company performed a complex and time-consuming consolidation procedure to build a centralized process repository. At $\mathrm{C} 4$, the tool is capable of exporting stored process models into a machine-readable file format. $\mathrm{C} 6, \mathrm{C} 7$, and $\mathrm{C} 8$ provide a central tool, which connects to the companies' IT infrastructure. It can perform basic conformance checks and prompts the user for additional data to structure the repository.

Almost all case companies confirm the benefits of a collaboration platform for process modeling. However, in most cases, platforms feature limited functionalities, including accessing and filtering process data. Only C6 uses a central platform that facilitates communication by offering sharing, ranking, and commenting functionalities. Today, the coordination of modeling is becoming increasingly independent, with employees highlighting issues and solving them collaboratively.

People. The area of people requires enterprises to implement easy-to-use methods and to provide training offers that build skills and foster process orientation.

All case companies confirm the importance of training offers. C6 provides a training program that consists of beginner courses on modeling basics and more specific formats on advanced topics. Similarly, C8 uses online courses to provide information on and solutions for organizational challenges. Furthermore, the company establishes a mentoring program for novice modelers and holds annual conferences, in which it communicates on developments and changes.
At all other companies, educational offers focus on establishing a basic understanding of modeling and on how it contributes to the success of other initiatives.

To foster usability, $\mathrm{C} 1$ and $\mathrm{C} 2$ focus on providing guidelines and conventions that reduce modeling complexity. Both companies further use structuration criteria to organize their process repositories and to accelerate query procedures. At $\mathrm{C} 2$ and $\mathrm{C} 7$, modelers emphasize the usefulness of comments and texts that augment process models. At C3, modelers specify the objectives linked to a modeling project and locate it within the company's process architecture. Furthermore, the tool queries the company's repository for similar process models, which are reusable as modeling templates. C6 confirms the importance of easy-to-use tools and specifications for the company's bottom-up approach. In fact, the company scrutinizes all specifications and functionalities toward their usability and adapts them if they yield stakeholder resistance. C8 further equips its modeling tool with functionalities to export process models into an ordered list of activities to improve their understandability.

Culture. The dimension of culture requires enterprises to facilitate process orientation.

All case companies confirm the importance of cultural aspects. $\mathrm{C} 1$ focuses on ensuring commitment by providing easy-to-use modeling specifications. $\mathrm{C} 1, \mathrm{C} 3$, $\mathrm{C} 4$, and $\mathrm{C} 8$ foster a common understanding of modeling and its relevancy for business success. Similar to its overall strategy, C6 follows a bottom-up approach and focuses on the viral diffusion and adoption of process modeling.

To enable process awareness, C2 implements a communication plan that continuously highlights the benefits of modeling for collaboration and organizational performance. $\mathrm{C} 1, \mathrm{C} 3$, and $\mathrm{C} 8$ further use process orientation as an organization-wide philosophy. Thus, the companies provide adequate incentives to build process awareness and to facilitate an understanding of their roles and responsibilities within the interconnected process structure. At C6, process orientation drives the bottom-up modeling approach. Initially, the company focused on establishing process awareness among key users, who then convinced other employees. Subsequently, the company's employees began to pick up the modeling task and started to fully commit to the initiative with a constantly high participation rate today.

\section{$5 \quad$ A Framework for Successful Process Modeling Initiatives}

Our results suggest that enterprises must carefully analyze their business needs to design successful 
modeling initiatives. We combine theoretical implications with insights from practice to answer RQ2. Hence, we extend our success factor model with additional parameters and construct a decision framework in Table 2. However, not all companies address each capability area in the same way. Instead, we notice that some capabilities are equally relevant, while the importance of others varies across companies. To account for these general and contextual components, we add a configuration feature to Table 2. General capabilities do not depend on a company's characteristics. For example, all companies highlight the importance of an integrated and easy-touse IT infrastructure, without linking such characteristics to a particular goal or initiative. We consider them as mandatory. By contrast, contextual capabilities demand a situational configuration. Companies must align them with their environment and with their objectives.

While contextual capabilities can vary across scenarios, our case companies choose from a fixed set of configuration opportunities that correlate strongly with their modeling objectives. Because all initiatives yield change and demand sufficient resources, they require management support. Although the top managements of all companies approve the initiatives, they adjust their commitment to the project's needs. In fact, top-management support can range from observing, to supporting, and involved. The companies choose between top-down and bottom-up approaches to control process modeling. While in top-down initiatives, modeling is performed by centralized process analysts, bottom-up approaches indicate distributed efforts at the place of process execution. Furthermore, hybrid approaches combine both designs and entail multiple distributed modeling projects aligned in and coordinated by a central instance.

Conventions and guidelines can support or regulate the modeling task. If quality is important, companies implement rigid conventions and guidelines. To ensure interoperability, they use restrictive specifications. Ultimately, they define guiding specifications to foster communication and collaboration.

The companies store process models in integrated process repositories. To ensure agility and accessibility, they further deploy measures to structure, manage, or control their repositories. In structured repositories, they define a set of rules and naming conventions to group and sort their processes. By contrast, managed repositories entail an active control that is performed after the model's introduction. In the case of a controlled repository, each model is checked against a set of requirements before its admission.

All companies use modeling software. However, tools differ regarding their capabilities for collaboration.
While individual tools focus on modeling exclusively, collaborative software also provides the means for communication and collaboration.

We identify three types of educational offers for process modeling. Targeted education focuses on a small number of key users. Selective education incorporates all stakeholders involved in process modeling. Ultimately, holistic approaches educate all stakeholders on process awareness and modeling basics. We summarize these configurations in Table 2 .

\section{$6 \quad$ Strategies for Successful Process Modeling Initiatives}

We can derive three strategies for successful modeling initiatives.

\subsection{Process Automation}

To accomplish the goal of process automation, enterprises must implement a strategy centered on process modeling quality. Supported by an involved top management, we recommend implementing a topdown managed governance, which defines rules, roles, and responsibilities centrally and monitors their compliance continuously. To ensure that process models are executable, we further suggest establishing a multi-stage reviewing process, which requires process analysts and software developers to crosscheck the models' quality at multiple times. Thus, enterprises should provide a set of rigid conventions and best practice models as blueprints.

However, as automation requires high-skilled process modelers, guidelines typically have an insignificant impact on modeling quality and can be limited to key aspects. To ensure consistency and to provide a single source of truth, we recommend enterprises to establish a controlled repository, which allows stakeholders to access and view process models.

Consequently, this entails that the central BPM team only can modify or add process models. As minor changes can affect the applicability of process models in automation environments, enterprises must implement a version-management system. To support the modeling task, they must further provide individual tools that perform quality checks at runtime. In addition, they rely on a seamlessly integrated IT infrastructure to increase the project's feasibility. Ultimately, enterprises rely on targeted education offers. These must include periodic training courses on advanced modeling topics, which are typically necessary after modifying modeling conventions or the software environment. 
Table 2. Recommendations for successful process modeling initiatives

\begin{tabular}{|c|c|c|c|}
\hline & Recommendation & Description & Configuration \\
\hline \multirow{5}{*}{ 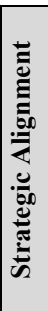 } & $\begin{array}{l}\text { Define clear goals for your process } \\
\text { modeling initiative. }\end{array}$ & $\begin{array}{l}\text { Process Modeling can be used to accomplish various goals, including documentation, standardization, and automation. While realizing all benefits at once seems } \\
\text { desirable, different goals require different strategies. Hence, enterprises must focus on the most promising goals and accomplish others in subsequent initiatives. }\end{array}$ & Contextual \\
\hline & Ensure top-management support. & $\begin{array}{l}\text { Management commitment is essential for process modeling success. Consider what type of management support is necessary to support your strategy. } \\
\text { Enterprises must identify, analyze, and estimate their initiative's needs for financial and other organizational resources. }\end{array}$ & Contextual \\
\hline & Develop a communication plan. & $\begin{array}{l}\text { As organizational improvements take time, the benefits of process modeling are realized in the long-term. Enterprises must deploy a detailed communication } \\
\text { plan that links their modeling initiative to their overall strategy in order to maintain stakeholder motivation. }\end{array}$ & General \\
\hline & \begin{tabular}{|l} 
Integrate your improvement \\
initiatives.
\end{tabular} & $\begin{array}{l}\text { Because enterprises operate in a dynamic environment, it is sometimes necessary to launch multiple improvement projects simultaneously. However, they must } \\
\text { align these initiatives to avoid opposing objectives and to ensure the efficient use of organizational resources. }\end{array}$ & General \\
\hline & Provide a process architecture. & $\begin{array}{l}\text { Processes constitute a complex organizational system with numerous interdependencies and weak ties. Enterprises must provide a process architecture to } \\
\text { improve decision making and to coordinate process modeling activities. }\end{array}$ & General \\
\hline \multirow{3}{*}{ 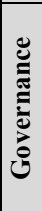 } & Define roles and responsibilities. & $\begin{array}{l}\text { Process modeling requires rules to ensure that the right things are done and that they are done in the right way. Enterprises must define a consistent system of } \\
\text { rules, roles, and responsibilities that fosters accountability and regulates decision making. }\end{array}$ & General \\
\hline & $\begin{array}{l}\text { Define conventions and guidelines to } \\
\text { support or regulate process modeling. }\end{array}$ & $\begin{array}{l}\text { Process models are critical to successful BPM initiatives, as they provide the basis for analysis and optimization. As modeling can be complex and time } \\
\text { consuming, its quality can greatly benefit from guidelines that support or regulate the task. }\end{array}$ & Contextual \\
\hline & $\begin{array}{l}\text { Define conventions and guidelines to } \\
\text { support or regulate process modeling. }\end{array}$ & $\begin{array}{l}\text { Conventions and guidelines can increase modeling quality, but they can also limit the expressiveness of resulting process models. Enterprises must define } \\
\text { conventions but provide the flexibility to capture different business scenarios. They should use guidelines to specify the scope of process modeling, but avoid } \\
\text { overregulation. Different modeling goals further require a different configuration. }\end{array}$ & Contextual \\
\hline \multirow{4}{*}{  } & $\begin{array}{l}\text { Select an adequate modeling } \\
\text { language. }\end{array}$ & $\begin{array}{l}\text { Enterprises must provide a modeling language to ensure model comparability and interoperability. They should modify the language only if adaptations yield } \\
\text { significant improvements or to comply with requirements in special business contexts. }\end{array}$ & General \\
\hline & Manage model versions. & $\begin{array}{l}\text { Previous model versions can uncover shifts in stakeholder and customer requirements. Enterprises should implement a version-management system to keep track } \\
\text { of these changes. }\end{array}$ & General \\
\hline & Manage model variants. & $\begin{array}{l}\text { While process models can foster communication, they can also be used as instructions during operative tasks. However, both use cases require different levels of } \\
\text { abstraction and detail. Enterprises should implement a variant-management system to address different information needs across different stakeholders and use } \\
\text { cases. }\end{array}$ & General \\
\hline & Facilitate stakeholder collaboration. & $\begin{array}{l}\text { In process-aware organizations, stakeholders uncover tacit process knowledge. Enterprises must implement a central platform to foster and support } \\
\text { communication and collaboration. }\end{array}$ & General \\
\hline \multirow[t]{2}{*}{$E$} & Provide tool support. & $\begin{array}{l}\text { Modeling tools can improve the effectiveness, efficiency, and quality of process modeling. However, as different tools can be used for different application } \\
\text { scenarios, enterprises must consider organizational requirements for tool selection. }\end{array}$ & Contextual \\
\hline & Integrate your IT infrastructure. & Process models can support other initiatives. Hence, enterprises must implement a central database that integrates with other tools to ensure interconnectivity. & General \\
\hline \multirow{2}{*}{$\frac{0}{\ddot{\Xi}}$} & Provide an education program. & $\begin{array}{l}\text { Well-trained modelers are essential for the success of process modeling initiatives. Enterprises must establish education programs that provide stakeholders with } \\
\text { a basic understanding of the modeling task and its organizational benefits. }\end{array}$ & Contextual \\
\hline & Ensure adequate ease of use. & $\begin{array}{l}\text { As process modeling can accomplish multiple objectives, its outcomes must be applicable and understandable. Hence, enterprises should keep their initiative's } \\
\text { setup simple, communicate actions clearly, and ensure tool accessibility and applicability. }\end{array}$ & General \\
\hline \multirow{2}{*}{ 这 } & Ensure stakeholder commitment. & $\begin{array}{l}\text { The success of process modeling depends on stakeholder participation. Enterprises must foster intuitiveness, highlight benefits, and align corresponding } \\
\text { procedures with organizational requirements to ensure stakeholder buy-in. }\end{array}$ & General \\
\hline & Facilitate process orientation. & $\begin{array}{l}\text { Process modeling builds upon process-aware employees. In functional organizations, process orientation does not emerge automatically. Instead, enterprises } \\
\text { must communicate its benefits and provide educational offers. }\end{array}$ & General \\
\hline
\end{tabular}




\subsection{Standardization}

Initiatives with the goal of standardization are typically less regulated. However, a supporting top management can improve the project's acceptance. As standardization requires process models' interoperability, we recommend providing restrictive conventions to break down business activities. However, process models are frequently used to guide operations and workflows. Hence, enterprises should avoid overregulation and ensure their expressiveness. Process modelers in standardization initiatives are typically skilled and experienced. Thus, enterprises can limit guidelines to advanced aspects, for example, on how to conduct interviews. They should further use a hybrid approach for data collection and governance. Thus, they can perform decision making centrally, while collecting information from distributed process stakeholders. Enterprises can foster the applicability of process models by providing a managed repository and a user-oriented variant-management system. While the repository enables stakeholders to quickly access information, variants allow them to adapt standardized process models to their individual needs. In addition, individual or collaborative tools must support the modeling task and connect with the enterprises' IT infrastructure. As the quality and coverage of modeling outcomes depends on stakeholder participation, enterprises rely on process awareness and commitment. Consequently, they must offer a selected education program and ensure the ease-of-use and intuitiveness of modeling outcomes.

\subsection{Communication}

A communication strategy builds upon decentralization and collaboration. As communication is most effective on operational levels, an observing top management should approve the project, without becoming actively involved. Yet, enterprises must define a comprehensive communication plan that summarizes the strategy and highlights potential benefits for individual stakeholders. They must also deploy a bottom-up approach that delegates decision making and accountability to a self-regulated social system. As the approach demands stakeholder participation, enterprises must define a set of guiding conventions without overregulating the modeling task. However, as modelers are typically low-skilled and unexperienced, enterprises must provide guidelines, best practices, and methodologies. Distributed modeling yields large amounts of process models. Hence, enterprises rely on structured repositories and version-management and variant-management systems that ensure consistency and avoid redundancies. To facilitate collaboration, enterprises must implement collaborative tools that are easily accessible and provide social networking functionalities to support communication. Ultimately, a communication strategy requires holistic education programs that continuously teach novices and experts on the basics of process modeling as well as on advanced topics.

\section{$7 \quad$ Conclusion and Limitations}

In this paper, we examined the requirements for successful process modeling initiatives from both a theoretical and an empirical perspective. First, we conducted a literature analysis to answer RQ1. We build upon an established framework to structure our findings and to construct a success factor model. To answer RQ2, we conducted eight case studies at companies that have introduced process modeling successfully. We analyzed how each company addressed the proposed success factors and merged our findings into a decision-support framework. Our results suggest that there is not a single success strategy for process modeling. Instead, enterprises must provide both general and contextual capabilities. To support enterprises in configuring contextual capabilities, we consolidated our findings in a morphological analysis and derived three high-level success strategies.

This research is not without limitations. Our success factor model stems from various literature findings. Despite applying a systematic search and analysis procedure, we cannot eliminate the possibility that we missed contributions that might have offered additional insights. We further applied a case study research design to analyze strategies to address modeling success factors in practice. As case study data provides only limited capabilities for statistical testing and validation, more empirical research is necessary to provide definite evidence. Analyzing case study data is further prone to subjective bias and depends on the researchers conducting it. Therefore, other researchers may have produced different results.

We did not account for the feasibility and exhaustiveness of the presented strategies. In some cases, operational constraints or setup costs can exceed benefits. In other cases, different configurations may also yield process modeling success. Nevertheless, we view these strategies as best practices that must to adapted to different business contexts. Furthermore, we did not examine the role and potential of process mining for successful modeling initiatives. Ultimately, we do not consider our modeling objectives as exhaustive. Instead, future research must evaluate how other objectives relate to this paper's recommendations. 


\section{$8 \quad$ References}

[1] M. Rosemann and J. vom Brocke, "The Six Core Elements of Business Process Management," in Handbook on Business Process Management 1: Introduction, Methods, and Information Systems, J. vom Brocke and M. Rosemann, Eds. Berlin: Springer, 2015, pp. 105-122.

[2] M. Indulska, P. Green, J. Recker, and M. Rosemann, "Business Process Modeling: Perceived Benefits," in International Conference on Conceptual Modeling, 2009, pp. 458-471.

[3] M. Dumas, M. La Rosa, J. Mendling, and H. A. Reijers, Fundamentals of Business Process Management. Berlin: Springer, 2013.

[4] J. Webster and R. T. Watson, "Analyzing the Past to Prepare for the Future: Writing a Literature Review," MIS Q., vol. 26, no. 2, pp. xiii-xxiii, 2002.

[5] M. Indulska, J. Recker, M. Rosemann, and P. Green, "Business Process Modeling: Current Issues and Future Challenges," 21st International Conference on Advanced Information Systems Engineering, pp. 501-514, 2009.

[6] R. K. Yin, Case Study Research: Design and Methods, vol. 5. Newbury Park, CA: Sage Publications, 1989.

[7] M. Röglinger, J. Pöppelbuß, and J. Becker, "Maturity Models in Business Process Management," Bus. Process Manag. J., vol. 18, no. 2, pp. 328-346.

[8] M. Rosemann, "Potential Pitfalls of Process Modeling: Part B," Bus. Process Manag. J., vol. 12, no. 3, pp. 377-383, 2006.

[9] P. Trkman, "The Critical Success Factors of Business Process Management," Int. J. Inf. Manage., vol. 30, no. 2, pp. 125-134, 2010.

[10] M. V. Drissen-Silva and R. J. Rabelo, "A Collaborative Decision Support Framework for Managing the Evolution of Virtual Enterprises,” Int. J. Prod. Res., vol. 47, no. 17,pp. 4833-4854, 2009.

[11] F. Milani, M. Dumas, N. Ahmed, and R. Matulevičius, "Modelling Families of Business Process Variants: A Decomposition Driven Method," Inf. Syst., vol. 56, pp. 55-72, 2016.

[12] I. Malavolta, P. Lago, H. Muccini, P. Pelliccione, and A. Tang, "What Industry Needs from Architectural Languages: A Survey," IEEE Trans. Softw. Eng., vol. 39, no. 6, pp. 869-891, 2013.

[13] M. Rosemann, "Potential Pitfalls of Process Modeling: Part A," Bus. Process Manag. J., vol. 12, no. 2, pp. 249-254, 2006.

[14] J. Recker, M. Rosemann, M. Indulska, and P. Green, "Business Process Modeling: A Comparative Analysis," J. Assoc. Inf. Syst., vol. 10, no. 4, pp. 333-363, 2009.

[15] M. B. Araújo and R. F. Gonçalves, "Selecting a Notation to Modeling Business Process: A Systematic Literature Review of Technics and Tools," in International Conference on Advances in Production Management Systems, 2016, pp. 198-205.
[16] P. F. Green and M. Rosemann, "Ontological Analysis of Integrated Process Modeling: Some Initial Insights," 11th Australasian Conference on Information Systems. Brisbane, 2000.

[17] J. Recker, M. Rosemann, P. Green, and M. Indulska, "Do Ontological Deficiencies in Modeling Grammars Matter?,"MIS Q., vol. 35, no. 1, pp. 57-79, 2011.

[18] J. Recker, "Opportunities and Constraints: The Current Struggle with BPMN," Bus. Process Manag. J., vol. 16, no. 1, pp. 181-201, 2010.

[19] N. Pflanzl and G. Vossen, "Challenges of Social Business Process Management," 47th Hawaii International Conference on System Sciences, pp. 3868-3877, 2014.

[20] A. Koschmider, M. Song, and H. A. Reijers, "Social Software for Business Process Modeling," J. Inf. Technol., vol. 25, no. 3, pp. 308-322, 2010.

[21] S. P. Gayialis, G. A. Papadopoulos, S. T. Ponis, P. Vassilakopoulou, and I. P. Tatsiopoulos, "Integrating Process Modeling and Simulation with Benchmarking using a Business Process Management System for Local Government," Int. J. Comput. Theory Eng., vol. 8, no. 6, p. 482, 2016.

[22] S. Suriadi, R. Andrews, A. H. M. ter Hofstede, and M. T. Wynn, "Event Log Imperfection Patterns for Process Mining: Towards a Systematic Approach to Cleaning Event Logs,” Inf. Syst., vol. 64, pp. 132-150, 2017.

[23] F. Imgrund, C. Janiesch, and C. Rosenkranz, "'Simply Modeling' - BPM for Everybody: Recommendations from the Viral Adoption of BPM at 1\&1," in Business Process Management Cases: Digital Innovation and Business Transformation in Practice, J. vom Brocke and J. Mendling, Eds. Heidelberg: Springer, 2017, pp. 521-540.

[24] S. Erol, M. Granitzer, S. Happ, S. Jantunen, B. Jennings, P. Johannesson, A. Koschmider, S. Nurcan, D. Rossi, and R. Schmidt, "Combining BPM and Social Software: Contradiction or Chance?,' J. Softw. Maint. Evol. Res. Pract., vol. 22, no. 6-7, pp. 449-476, 2010.

[25] G. Bruno, F. Dengler, B. Jennings, R. Khalaf, S. Nurcan, M. Prilla, M. Sarini, R. Schmidt, and R. Silva, "Key Challenges for Enabling Agile BPM with Social Software," J. Softw. Maint. Evol. Res. Pract., vol. 23, pp. 297-326, 2011.

[26] V. Gokaldas and M. E. Rangiha, "A Framework for Improving User Engagement in Social BPM," in Business Process Management Workshops, 2018, pp. 391-402.

[27] M. Zimoch, T. Mohring, R. Pryss, T. Probst, W. Schlee, and M. Reichert, "Using Insights from Cognitive Neuroscience to Investigate the Effects of Event-Driven Process Chains on Process Model Comprehension," in BPM Workshops, 2018, pp. 446-459.

[28] J. Mendling, J. Recker, H. A. Reijers, and H. Leopold, "An Empirical Review of the Connection Between Model Viewer Characteristics and the Comprehension of Conceptual Process Models," Inf. Syst. Front., 2018.

[29] W. Bandara, G. G. Gable, and M. Rosemann, "Factors and Measures of Business Process Modelling: Model Building Through a Multiple Case Study," Eur. J. Inf. Syst., vol. 14, no. 4, pp. 347-360, 2005. 